\title{
New measurements of muon decay from $\mathcal{T} \mathcal{W} \mathcal{I} \mathcal{T}$
}

\author{
G. M. Marshall, on behalf of the $\mathcal{T} \mathcal{W I S T}$ Collaboration ${ }^{\dagger}$ \\ TRIUMF, 4004 Wesbrook Mall, Vancouver, BC V6T 2A3 Canada
}

\begin{abstract}
The TRIUMF Weak Interaction Symmetry Test $(\mathcal{T} \mathcal{W} \mathcal{I} \mathcal{T})$ has recently completed its first physics analyses. The results represent a significant increase in precision for two of the four Michel parameters describing the energy and angle distributions of positrons from polarized positive muon decay. This is the first step toward our eventual goal of improving upon previous determinations for three of the parameters by at least an order of magnitude, as a test of the Standard Model in the purely leptonic decay interaction. $\mathcal{T} \mathcal{W} \mathcal{I} \mathcal{T}$ uses a polarized muon beam stopping at the center of a spectrometer consisting of a low mass, high precision array of planar drift chambers in a two tesla solenoidal field. The talk will focus on the operation of the device and the methods which are used to extract the decay parameters in a reliable way. Systematic uncertainties are especially important, as they will limit the final results.
\end{abstract}

\section{Introduction}

$\mathcal{T} \mathcal{W} \mathcal{I} \mathcal{T}$, the TRIUMF Weak Interaction Symmetry Test, is in the process of measuring the parameters describing the angle (with respect to muon spin) and energy distributions of positrons $\left(e^{+}\right)$from positive muon $\left(\mu^{+}\right)$decay. These muon decay parameters, or Michel parameters (after Prof. L. Michel [1]), offer a compact and convenient prediction of the

* email: glen.marshall@triumf.ca

$\dagger$ http://twist.triumf.ca/: R. Bayes, Yu.I. Davydov, J. Doornbos, W. Faszer, M.C. Fujiwara, D.R. Gill, P. Gumplinger, R.S. Henderson, J. Hu, J.A. Macdonald, G.M. Marshall, R.E. Mischke, M. Nozar, K. Olchanski, A. Olin, R. Openshaw, T.A. Porcelli, J.-M. Poutissou, R. Poutissou, G. Sheffer, B. Shin (TRIUMF), A. Gaponenko, P. Kitching, R.P. MacDonald, M.A. Quraan, N.L. Rodning, J. Schaapman, G. Stinson (University of Alberta), M.D. Hasinoff, B. Jamieson (University of British Columbia), P. Depommier (Université de Montréal), E.W. Mathie, R. Tacik (University of Regina), V. Selivanov, V.D. Torokhov (Russian Research Center, Kurchatov Institute), C.A. Gagliardi, J.R. Musser, R.E. Tribble, M. Vasiliev (Texas A\&M), D.D. Koetke, P.M. Nord, T.D.S. Stanislaus (Valparaiso University). 
electroweak interaction in muon decay. They can be related to a more recent description in terms of coupling constants for interactions of muons and electrons of definite handedness $[2]$.

The results reviewed here represent an increase in the experimental precision for the $\rho$ [3] and $\delta[4]$ parameters by factors of 2.5 and 2.9 respectively. We are in the process of further improving these results at the same time as we make our first direct measurement of $P_{\mu} \xi$. The eventual goal of $\mathcal{T} \mathcal{W I S T}$ is to improve all three parameters by at least one order of magnitude compared with previous experiments.

\section{Physics motivation}

The Standard Model of the strong, weak and electromagnetic interactions, based on the gauge group $S U(3)_{C} \times S U(2)_{L} \times U(1)_{Y}$, has proven to be remarkably successful in describing the existing experimental observations. At present, there exist no experimental results that deviate from its expectations, with the exception of neutrino oscillations which in any case result from a simple extension to the mass matrices. However, the Standard Model is universally believed to be an incomplete theory of nature in spite of its many successes, and many extensions have been proposed.

Normal muon decay $(\mu \rightarrow e \nu \bar{\nu})$ is a process which is exceptionally well suited to studies of the space-time structure of the weak interaction. This comes about because the purely leptonic nature of the decay eliminates many uncertainties due to internal structure of the particles or to contributions from other interactions.

By assuming a completely general, local, lepton-number-conserving, four-fermion point interaction, the matrix element for muon decay can be written in the charge-changing form as $[2,5]$ :

$$
M=\frac{4 G_{F}}{\sqrt{2}} \sum_{\substack{\gamma=S, V, T \\ \varepsilon, \mu=R, L}} g_{\varepsilon \mu}^{\gamma}\left\langle\bar{e}_{\varepsilon}\left|\Gamma^{\gamma}\right|\left(\nu_{e}\right)_{n}\right\rangle\left\langle\left(\bar{\nu}_{\mu}\right)_{m}\left|\Gamma_{\gamma}\right| \mu_{\mu}\right\rangle
$$

This includes scalar, vector, and tensor $\left(\Gamma^{S}, \Gamma^{V}, \Gamma^{T}\right)$ interactions among charged lepton spinors of definite chirality $(\varepsilon, \mu=R$ or $L)$. There are 10 complex amplitudes $g_{\varepsilon \mu}^{\gamma}\left(g_{L L}^{T}\right.$ and $g_{R R}^{T}$ are zero), resulting in 19 independent real parameters. In the Standard Model, $g_{L L}^{V}=1$ and all others are zero (a $V-A$ interaction). Constraints on the values of the coupling constants are derived from observables.

Under the same assumptions, neglecting neutrino masses and radiative corrections and averaging over the polarization of the decay $e^{+}$, the differential decay rate of the positive muon is expressed as $[1,6,7]$

$$
\frac{d^{2} \Gamma}{d x d \cos \theta}=\frac{1}{4} m_{\mu} W_{\mu e}^{4} G_{F}^{2} \sqrt{x^{2}-x_{0}^{2}}\left\{\mathcal{F}_{I S}(x)+\mathcal{P}_{\mu} \cos \theta \cdot \mathcal{F}_{A S}(x)\right\}
$$


where

$$
\begin{aligned}
W_{\mu e} & =\frac{m_{\mu}^{2}+m_{e}^{2}}{2 m_{\mu}} \\
x & =\frac{E_{e}}{W_{\mu e}} \\
x_{0} & =\frac{m_{e}}{W_{\mu e}}
\end{aligned}
$$

$$
\begin{aligned}
\mathcal{P}_{\mu} & =\left|\overrightarrow{\mathcal{P}}_{\mu}\right| \\
\cos \theta & =\frac{\overrightarrow{\mathcal{P}}_{\mu} \cdot \vec{p}_{e}}{\left|\overrightarrow{\mathcal{P}}_{\mu}\right|\left|\vec{p}_{e}\right|}
\end{aligned}
$$

The isotropic and asymmetric parts, respectively, in the decay rate are written in terms of the decay (Michel) parameters $\rho, \eta, \delta$, and $\xi$.

$$
\begin{aligned}
\mathcal{F}_{I S}(x) & =x(1-x)+\frac{2}{9} \rho\left(4 x^{2}-3 x-x_{0}^{2}\right)+\eta x_{0}(1-x) \\
\mathcal{F}_{A S}(x) & =\frac{1}{3} \xi \sqrt{x^{2}-x_{0}^{2}}\left[1-x+\frac{2}{3} \delta\left\{4 x-3+\left(\sqrt{1-x_{0}^{2}}-1\right)\right\}\right]
\end{aligned}
$$

Radiative corrections to the distribution are substantial, and must be incorporated. Corrections have been calculated including full $O(\alpha)$ radiative corrections with exact electron mass dependence, leading and next-to-leading logarithmic terms of $O\left(\alpha^{2}\right)$, leading logarithmic terms of $O\left(\alpha^{3}\right)$, corrections for soft pairs, virtual pairs, and an ad-hoc exponentiation $[8,9,10]$. The result is adequate for measurements with a precision better than $10^{-3}$, and higher order estimates, motivated by the sensitivity of $\mathcal{T} \mathcal{W} \mathcal{I S T}$, have recently been reported [11].

The Standard Model predicts the decay parameters to take the values $\rho=\frac{3}{4}, \eta=0, \delta=\frac{3}{4}$, and $\xi=1$. The actual values of the parameters are very sensitive to the Standard Model assumption that the interaction is pure left-handed vector $(V-A)$; if terms with other characteristics are present, the parameters differ. These decay parameters, and combinations of them, can be written in terms of bilinear combinations of the more general and fundamental coupling constants $g_{\varepsilon \mu}^{\gamma}$, such that deviations from Standard Model values will place very model independent limits on the individual couplings.

Fetscher et al. [2] expressed the relationship in terms of probabilities $Q_{\varepsilon \mu}(\varepsilon, \mu=R, L)$ for a $\mu$ handed muon to decay into a $\varepsilon$ handed electron:

$$
Q_{\varepsilon \mu}=\frac{1}{4}\left|g_{\varepsilon \mu}^{S}\right|^{2}+\left|g_{\varepsilon \mu}^{V}\right|^{2}+3\left(1-\delta_{\varepsilon \mu}\right)\left|g_{\varepsilon \mu}^{T}\right|^{2}
$$

To demonstrate how the Michel parameters can be used in a model independent test for handedness of the muon coupling, the probability for a right-handed muon coupling can be written as:

$$
\begin{aligned}
Q_{R}^{\mu} & \equiv Q_{R R}+Q_{L R} \\
& =\frac{1}{4}\left|g_{L R}^{S}\right|^{2}+\frac{1}{4}\left|g_{R R}^{S}\right|^{2}+\left|g_{L R}^{V}\right|^{2}+\left|g_{R R}^{V}\right|^{2}+3\left|g_{L R}^{T}\right|^{2} \\
& =\frac{1}{2}\left[1+\frac{1}{3} \xi-\frac{16}{9} \xi \delta\right]
\end{aligned}
$$

The Review of Particle Physics [5] summarizes the best measurements prior to $\mathcal{T} \mathcal{W I S T}$ : 


$$
\begin{aligned}
\rho & =0.7518 \pm 0.0026 \\
\eta & =-0.007 \pm 0.013 \\
\delta & =0.7486 \pm 0.0026 \pm 0.0028 \\
\mathcal{P}_{\mu} \xi & =1.0027 \pm 0.0079 \pm 0.0030 \\
\mathcal{P}_{\mu} \frac{\xi \delta}{\rho} & >0.99682
\end{aligned}
$$

The first publications from $\mathcal{T} \mathcal{W} \mathcal{I} \mathcal{S}[3,4]$ reduce the uncertainties in $\rho$ and $\delta$ by factors of 2.5 and 2.9 respectively. In combination with Eq. 11 and the positive semidefinite probability of Eq. 6, the value of $\mathcal{P}_{\mu} \xi$ is significantly constrained as well. The main goal of $\mathcal{T} \mathcal{W I S T}$ is to search for new physics through more precise measurements of the muon decay parameters. We aim to set new limits on the right-handed coupling of the muon in a model independent way, as well as to squeeze the parameter space for classes of extensions to the Standard Model, such as those invoking left-right symmetry, with less model dependence than otherwise possible.

Left-right symmetric (LRS) models include a heavier partner to the standard $W$ boson which has right handed coupling. Mixing of the two bosons $W_{1} \approx W_{L}$ and $W_{2} \approx W_{R}$ of mass $M_{1}$ and $M_{2}$, with angle $\zeta$, leads to small deviations of $\rho$ and $\xi$ from the Standard Model values. For example, a particularly simple version of an LRS model suggests

$$
\begin{aligned}
\frac{3}{4}-\rho & =\frac{3}{2} \zeta^{2} \\
\frac{1-P_{\mu} \xi}{4} & =\zeta^{2}+\frac{M_{1}^{4}}{M_{2}^{4}}+\zeta \frac{M_{1}^{2}}{M_{2}^{2}}
\end{aligned}
$$

A model which incorporates a nonlocal tensor interaction, motivated in part by results on weak radiative pion decay, leads to a modification of the Michel form and an independent parameter, $\kappa[12]$. If such a parameter was not zero, the effect on the decay spectrum shape would be a correlation with $\delta$ such that $\delta=\frac{3}{4}\left(1-6 \kappa^{2}\right)$.

\section{The experiment}

\subsection{The detector array}

The $\mathcal{T} \mathcal{W} \mathcal{I} \mathcal{S}$ detector (Fig. 1) is described in detail in [13]. It consists of a symmetric array of planar detectors, called the detector stack, which is constructed to very high precision with attention paid to minimizing the amount of material in the tracking region. It consists of 56 low-mass high-precision planar wire chambers. $\mathcal{T} \mathcal{W} \mathcal{I} \mathcal{S} \mathcal{T}$ uses two types of wire chamber detectors, which we call DCs and PCs; both types measure either the $u$ or $v$ coordinate, in a system rotated by $\pi / 4$ from the horizontal-vertical $(x, y)$ system. The DCs provide precise spatial information for track reconstruction, while the PCs offer better time resolution essential for event classification and track identification. Planes are positioned with relative accuracy of $5 \times 10^{-5}$ in the $z$ (beam and magnetic field) direction, using specially constructed glass ceramic spacers. Alignment in the transverse $u, v$ directions is accomplished with fits of straight tracks, but depends also on the wire separation accuracy which was measured with rms value of $\sigma=3.3 \mu \mathrm{m}$ for 6,304 sense wires in the chamber modules (including spare modules).

At the center of the array is a thin target in which the muons stop. The detector allows very precise measurement of positron decay tracks symmetrically, in forward and backward directions, 


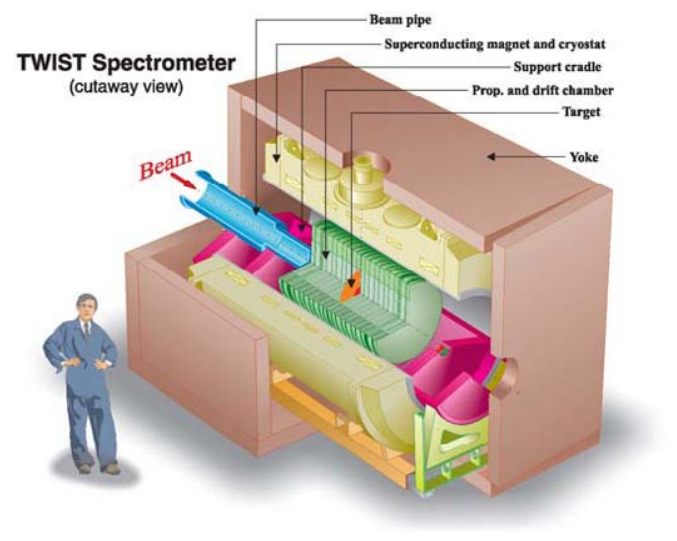

Figure 1: The detector arrangement within the superconducting solenoid.

while minimizing interactions which would broaden and distort the detector response function. The planar geometry means that the energy loss, which depends on the length of a track in any material in its path, has primarily a simple $1 /|\cos \theta|$ dependency.

\subsection{Solenoidal field}

The detector stack is in a very uniform solenoidal field of $2 \mathrm{~T}$, produced by a superconducting solenoid which was originally constructed about twenty years ago as an early-generation whole body MRI field device. The cryostat vacuum enclosure has an inner diameter of $100 \mathrm{~cm}$ and a length of $223 \mathrm{~cm}$. An external steel yoke was fabricated to produce the required field uniformity for the detector tracking volume. It also functions to contain the return field, reducing its effect on nearby people and equipment while also reducing the influence of external changes such as the position of the building crane on the internal field. The yoke was modeled with OPERA-3d prior to fabrication. It is $20 \mathrm{~cm}$ thick at the top and sides, and $8 \mathrm{~cm}$ thick at the ends. The downstream end of the yoke is hinged for easy installation and removal of the detector cradle. Within the tracking volume $(|z|<500 \mathrm{~mm}, r<160 \mathrm{~mm})$, field map measurements determine the variations of the field as a function of position to $5 \times 10^{-5}$. The field is uniform over the full volume to $4 \times 10^{-3}$ (full width); however, non-uniform field maps of precision approaching the measured maps, obtained by matching the measurements to an OPERA-3d calculation, are used for analysis and simulations. The field has also been mapped at $1.96 \mathrm{~T}$ and $2.04 \mathrm{~T}$.

\subsection{The muon beam}

A thin scintillator records the incoming muon, providing an unbiased trigger for events. The incident beam characteristics and the beam entrance path are engineered so as to minimize depolarization, the knowledge of which is crucial in setting limits on $\mathcal{P}_{\mu} \xi$.

Muons for $\mathcal{T} \mathcal{W I S T}$ are produced from positive pions $\left(\pi^{+}\right)$decaying at rest, at or near the surface of the pion production target (surface muons). For a massless left-handed neutrino, the absence of non-Standard Model interactions leads to a positive muon $\left(\mu^{+}\right)$with spin antiparallel 
to the momentum direction. The result is that surface muons have a high polarization in a direction opposite to the beam direction. They can be distinguished from so-called cloud muons of lower (and opposite) polarization using time-of-flight separation with respect to the convenient $43 \mathrm{~ns} \mathrm{RF}$ period of the accelerator.

Several factors contribute to a difference of the polarization from the ideal value. Most are controlled via a high quality, low emittance beam transport system, the M13 beam line, from the pion production target to the $\mathcal{T} \mathcal{W} \mathcal{I} \mathcal{S} \mathcal{T}$ solenoid entrance. The resulting beam consists of about $2.5 \times 10^{3}$ muons per second at about $29.6 \mathrm{MeV} / \mathrm{c}$, where the muon range is only approximately $1.5 \mathrm{~mm}$ in materials of density near one.

First of all, scattering of muons changes the momentum direction but not the spin direction, which leads to depolarization of the beam. A small and well-characterized emittance, with a narrow momentum variation as close as possible to the maximum surface muon value, make this depolarization small and easily estimated. Second, depolarization may also occur in the interactions of muons during and/or after thermalization in the muon stopping target or nearby materials. The muon stopping target also functions as the cathode for PCs on either side, so that muons stopping in the target can be identified with little ambiguity. From the positron distributions for decays after $1 \mu \mathrm{s}$ in the graphite-coated Mylar $(125 \mu \mathrm{m})$ target which was used for data taking in $2002[3,4]$, the mean initial polarization was estimated to be $93 \%$, with an exponential relaxation parameter of $0.0172(5) \mu \mathrm{s}^{-1}$. The depolarization was too high for the results to be useful in any direct measurement of $P_{\mu} \xi$. The subsequent use of a high purity aluminum $(71 \mu \mathrm{m})$ target, for data which is still under analysis, shows extremely small timedependent depolarization. A third significant potential source of depolarization for $\mathcal{T} \mathcal{W I S T}$ is the effect of the fringe field as the muon enters the solenoid, which is minimized by detailed knowledge of the fringe field distribution and the muon path through it. A low-mass, lowpressure beam measurement device (TEC) has recently been commissioned and used to record very precise information on the muon beam spatial and angular distributions in only a few minutes. We optimize those characteristics, measure them, and monitor them for long term stability in short dedicated periods interspersed with longer data sets without the TEC in place.

A gas degrader of length $20 \mathrm{~cm}$ is in the muon path inside the solenoid, after a vacuum isolation window and immediately before the beam passes through the trigger scintillator and enters the detector stack. A variable mixture of $\mathrm{He}$ and $\mathrm{CO}_{2}$ is automatically adjusted so that the muon stopping position as measured by the detector planes is in the correct place, to compensate for atmospheric density variations and thereby reduce a significant systematic uncertainty.

\section{Analysis}

The challenge in the analysis is to select the $e^{+}$emitted from $\mu^{+}$decays over the largest possible range of momentum and angle without introducing significant bias. The positron energy is calibrated at the kinematic end point at $52.8 \mathrm{MeV} / \mathrm{c}$. The analysis is applied both to the data and the simulation and the results are compared to determine the decay spectrum. Biases in the analysis are then compensated to the extent to which the simulation accurately reproduces the data. The validation of the simulation determines the associated systematic uncertainty.

The GEANT3 simulation contains virtually all detector components, and simulates detector response in detail, including ionization clusters. It uses a realistic beam profile based on measurements, and includes extra (pileup) muon and beam positron contamination. The output is in digitized format, identical in appearance to the real data. 


\subsection{Extraction of decay parameters and blind analysis}

To extract decay parameters from the experimental decay positron energy and angle spectrum, one needs to take into account detector response. This is accomplished by comparison with the GEANT3 Monte Carlo (MC), which simulates the response to a degree which can be independently verified. However, a simulation requires not just the theoretical formula, but also a set of specific values of decay parameters to proceed, and the result is a convolution of decay and detector response for only those values. To perform a fit for non-specific values of decay parameters, $\mathcal{T} \mathcal{W} \mathcal{I} \mathcal{T}$ has developed a technique which uses an expansion of the convoluted spectrum in deviations of the parameters from their MC values [14]. The scheme can be visualized by

$$
n_{i}\left(\alpha_{\text {Data }}\right)=n_{i}\left(\alpha_{\mathrm{MC}}\right)+\frac{\partial n_{i}}{\partial \alpha}\left(\alpha_{\text {Data }}-\alpha_{\mathrm{MC}}\right),
$$

where $n_{i}\left(\alpha_{\text {Data }}\right)$ is the experimental spectrum, $n_{i}\left(\alpha_{\mathrm{MC}}\right)$ is the simulated one, and $\Delta \alpha=\alpha_{\text {Data }}-$ $\alpha_{\mathrm{MC}}$ is the fit parameter. The $\partial n_{i} / \partial \alpha$ coefficient is calculated by Monte Carlo using analytical derivatives of the theoretical spectrum, where $\alpha$ stands for the decay parameters $\{\rho, \eta, \xi, \xi \delta\}$. Since the Michel form of the spectrum is linear in $\alpha$, the first-order expansion is exact in this parametrization.

The fitting procedure described above allows for a straightforward "blinding" of the analysis: since the fit gives only the deviations from the MC values, hiding $\alpha_{\mathrm{MC}}$ is sufficient. Values of decay parameters for $\mathrm{MC}$ production are generated randomly, within given tolerances, then hidden in encrypted form. They are used to produce a large set of "decays" $\{E, \cos (\theta)\}$ which is stored on disk and can be fed to GEANT.

The fitting procedure is also applied to the estimation of biases and systematic effects, where differences in decay parameters are determined from comparisons of data sets (real data or simulation) obtained under differing controlled conditions. Hidden parameters are revealed only after the full analysis and specification of systematic uncertainties.

\section{Systematic uncertainties}

The general method to evaluate systematic uncertainties is to exaggerate, in data and/or simulation, the underlying condition (e.g., a variation in detector high voltage can cause a change in efficiency or position-to-drift-time characteristics) beyond what would be expected in practice. The effect on each decay parameter can be extracted by fitting distributions corresponding to exaggerated and normal conditions, and the results scaled to the assessed real variation in data. It is important to pay careful attention to the possibilities of non-linearity in the exaggeration, or double counting of systematic effects (e.g., in the high voltage example, both efficiency and detector response are altered).

Sources of systematic uncertainties which have been considered are grouped into classifications as follows

Positron interactions: energy smearing, multiple scattering, hard interactions, interactions in materials both inside and outside the detector.

Chamber response: DC and PC efficiencies, chamber dead zones following arrival of a highly ionizing muon, long drift time corrections, voltage variations, temperature, pressure, chamber foil geometry (bulges), crosstalk, time zero variations.

Momentum calibration: end point fits, magnetic field reproduction. 
Muon beam stability: muon stopping location, beam intensity variations, and beam line magnet stability.

Spectrometer alignment: translational, rotational, longitudinal, field to detector axis alignments.

A summary of contributions to systematic uncertainties of $\rho$ and $\delta$ is shown in Table 1 .

Table 1: Contributions to the systematic uncertainty in $\rho$ and $\delta$. Average values are given for those denoted (ave), which are considered set dependent when performing the weighted average of the data sets.

\begin{tabular}{lll}
\hline Effect & Uncertainty in $\rho$ & Uncertainty in $\delta$ \\
\hline Chamber response (ave) & \pm 0.00051 & \pm 0.00056 \\
Stopping target thickness & \pm 0.00049 & \pm 0.00037 \\
Positron interactions & \pm 0.00046 & \pm 0.00055 \\
Spectrometer alignment & \pm 0.00022 & \pm 0.00061 \\
Momentum calibration (ave) & \pm 0.00020 & \pm 0.00029 \\
Theoretical radiative corrections & \pm 0.00020 & \pm 0.00010 \\
Track selection algorithm & \pm 0.00011 & \\
Muon beam stability (ave) & \pm 0.00004 & \pm 0.00010 \\
Upstream/downstream efficiencies & & \pm 0.00004 \\
\hline
\end{tabular}

Since $\mathcal{T} \mathcal{W} \mathcal{I} \mathcal{T}$ relies on a fit to a simulation, it is extremely important to verify the validity of the simulation. The validation process depends on the particular source of systematic uncertainty, including wherever possible a comparison with measurements; the measurements must not, of course, depend in any direct or precise way on the muon decay parameter values. One example which tests the critical assumptions of positron interactions as simulated by the GEANT3 code is to compare the measurements with fits to tracks predicted by the MC for the case where muons do not stop in the muon stopping target at the center of the detector stack, but rather, at one end of the detector stack. The resulting decay positrons can pass through both halves of the stack, on either side of the stopping target, where on one side the direction of the positron is opposite to that in normal events. This provides, for both MC and real data, two measurements of the same track. The differences in fitted energy and in angle in the two halves can be compared, and the result used to set a limit on several of the positron interaction systematics.

\section{Results}

A detailed presentation of analysis procedures and results can be found in $[3,4]$. To summarize, we find $\rho=0.75080 \pm 0.00044$ (stat.) \pm 0.00093 (syst.) \pm 0.00023 , consistent with the Standard Model expectation $\rho=3 / 4$. This result assumes that $\eta$ is given by the accepted value [5], $\eta=-0.007 \pm 0.013$; the third uncertainty represents the change in $\rho$ when $\eta$ changes within its uncertainty. Within left-right symmetric models, this result sets a new upper limit, $|\zeta|<0.030$ 


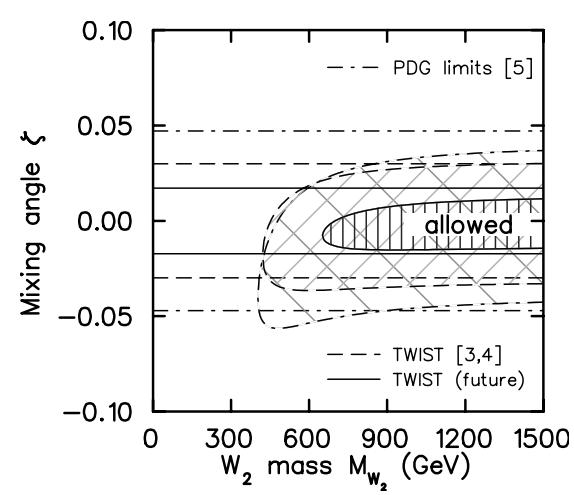

Figure 2: Limits from muon decay on parameters of the LRS model (see text).

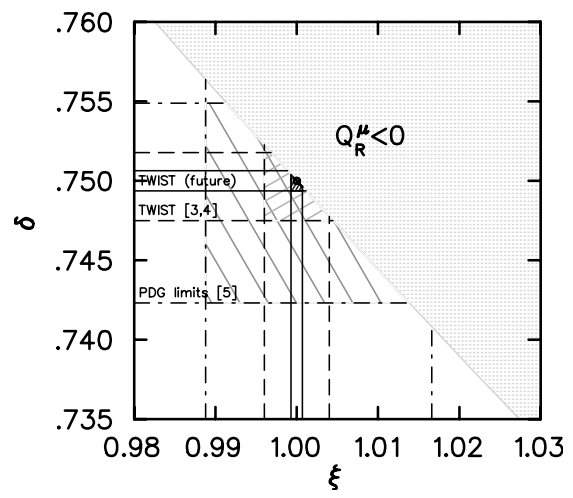

Figure 3: Limits of $\xi$ and $\delta$ and possible right-handed coupling of the muon (see text).

(90\% c.l.), on the $W_{L}-W_{R}$ mixing angle, as shown by the dashed horizontal lines in Fig. 2. In addition, we find $\delta=0.74964 \pm 0.00066$ (stat.) \pm 0.00112 (syst.), consistent with the Standard Model expectation of $3 / 4$. Using this along with the value for $\rho$, a previous measurement of $P_{\mu} \xi \delta / \rho[15]$, and the constraint $Q_{R}^{\mu} \geq 0$, it is possible to establish new $90 \%$ confidence interval limits, $0.9960<P_{\mu} \xi \leq \xi<1.0040$, consistent with the Standard Model value of 1 . This result is more restrictive than the current best measurements for muons from pion and kaon decays $[16,17]$. In addition, from these same results one finds that $Q_{R}^{\mu}<0.00184$ with $90 \%$ confidence. The previous, current, and potential future limits on $\delta$ and $\xi$ are plotted in Fig. 3 to show the extent to which a muon right-handed coupling is restricted. Regions are shown which correspond to prior (dot-dashed lines, coarse hatching), the current $\mathcal{T} \mathcal{W} \mathcal{I} \mathcal{S}$ measurement (dashed lines, medium), and future $\mathcal{T} \mathcal{W I S T}$ (solid lines, fine) measurements of muon decay. The shaded region is forbidden. The results may be combined with Eq. (6) to find new $90 \%$ confidence limits on interactions that couple right-handed muons to left-handed electrons: $\left|g_{L R}^{S}\right|<0.086$, $\left|g_{L R}^{V}\right|<0.043$, and $\left|g_{L R}^{T}\right|<0.025$.

The lower limit, $0.9960<P_{\mu} \xi$ can be used to determine a new limit on the mass of the possible right-handed boson, $W_{1} \approx W_{R}$, improving the existing lower limit of $406 \mathrm{GeV} / \mathrm{c}^{2}$ (402 $\mathrm{GeV} / \mathrm{c}^{2}$ with modern $\mathrm{M}_{W_{1}}=80.423 \mathrm{GeV} / \mathrm{c}^{2}$ ) from [15] to $420 \mathrm{GeV} / \mathrm{c}^{2}$ (90\% confidence) under the assumption of pseudo-manifest left-right symmetry. For nonmanifest left-right symmetric models the limit is $M_{W_{2}} g_{L} / g_{R}>380 \mathrm{GeV} / \mathrm{c}^{2}$, where $g_{L}$ and $g_{R}$ are the coupling constants [18]. Eventually, $\mathcal{T} \mathcal{W I S T}$ hopes to increase these two lower limits to 650 and $590 \mathrm{GeV} / \mathrm{c}^{2}$ respectively, with a direct measurement of $P_{\mu} \xi$ of precision less than $5 \times 10^{-4}$. The relative restrictions imposed by muon decay on the pseudo-manifest LRS parameters are shown in Fig. 2. Regions are shown which correspond to the prior best muon decay limit from $P_{\mu} \xi \delta / \rho$ [15] (coarse hatching, dotdashed), the current indirect $\mathcal{T} \mathcal{W} \mathcal{I S T} P_{\mu} \xi$ measurement (medium, dashed), and expected future direct $\mathcal{T} \mathcal{W} \mathcal{I S T} P_{\mu} \xi$ (fine, solid) measurements of muon decay. It should be noted that many other measurements, from nuclear $\beta$ decay to direct searches for a heavy $W$, provide restrictions on the LRS parameters; they are so numerous that it is impossible to summarize them in the figure. However, if the right-handed neutrino is light enough to be produced, muon decay is 
particularly insensitive to assumptions about the details of the LRS model, in particular the structure of the right-handed CKM matrix, since it is almost purely leptonic (muon polarization in pion decay provides the very weak dependence on the assumptions).

Finally, the value of $\delta$ is sensitive to a proposed nonlocal interaction [12] that would be represented by a new parameter $\kappa$. A limit for $\kappa$ may be estimated from our $90 \%$ confidence lower limit for $\delta$ using the relation $\delta=3 / 4\left(1-6 \kappa^{2}\right)$. This results in $\kappa \leq 0.024$, which compares with $\kappa=0.013[12]$ hinted at by $\pi$ decay experiments.

\section{Summary}

$\mathcal{T} \mathcal{W} \mathcal{I} \mathcal{T}$ has produced its first results for $\rho$ and $\delta$, both of which are consistent with Standard Model values. Data are being analyzed for our first direct measurement of $P_{\mu} \xi$, and improvements to all three parameters are foreseen. The ultimate precision will be limited by systematic uncertainties, in some cases by how well they can be determined from dedicated and specialized data sets. The dominant systematics are expected to arise from chamber response and positron interaction sources, and for the polarization measurement, the degree to which depolarization can be estimated.

This work was supported by funding from NSERC (Canada) and DOE (USA). Computing facilities of WestGrid are gratefully acknowledged.

\section{References}

[1] L. Michel, Proc. Phys. Soc. A63, 514 (1950).

[2] W. Fetscher, H.-J. Gerber, and K. F. Johnson, Phys. Lett. B173, 102 (1986).

[3] J. R. Musser et al., Phys. Rev. Lett. 94, 101805 (2005); arXiv:hep-ex/0409063 (TRI-PP04-21).

[4] A. Gaponenko et al., Phys. Rev. D71, 071101(R) (2005); arXiv:hep-ex/0410045 (TRI-PP04-22).

[5] S. Eidelman et al., Phys. Lett. B592, 1 (2004).

[6] C. Bouchiat and L. Michel, Phys. Rev. 106, 170 (1957).

[7] A. Sirlin, Phys. Rev. 108, 844 (1957).

[8] A. B. Arbuzov, Phys. Lett. B524, 99 (2002).

[9] A. Arbuzov, A. Czarnecki, and A. Gaponenko, Phys. Rev. D65, 113006 (2002).

[10] A. Arbuzov and K. Melnikov, Phys. Rev. D66, 093003 (2002).

[11] C. Anastasiou, K. Melnikov, and F. Petriello, arXiv:hep-ph/0505069.

[12] M. V. Chizhov, arXiv:hep-ph/0405073, 2004.

[13] R. L. Henderson et al., arXiv:hep-ex/0409066 (TRI-PP-04-20), accepted for publication in Nucl. Instr. and Meth., February 2005.

[14] A. Gaponenko, Ph.D. thesis, University of Alberta, 2005.

[15] A. Jodidio et al., Phys. Rev. D 34, 1967 (1986); 37, 237(E) (1988).

[16] I. Beltrami et al., Phys. Lett. B194, 326 (1987).

[17] J. Imazato et al., Phys. Rev. Lett. 69, 877 (1992).

[18] P. Herczeg, Phys. Rev. D34, 3449 (1986). 